\title{
Vaginocutaneous fistula and inguinal abcess presented 6 years after tension-free vaginal tape sling
}

\author{
Ali Feyzullah Şahin ${ }^{1}$, Yusuf Özlem İlbey ${ }^{2}$, Nur Şahin ${ }^{3}$ \\ ${ }^{1}$ Assistant Professor in Urology - Department of Urology, Şifa University Medicine School, İzmir, Turkey; \\ 2 Associative Professor in Urology - Department of Urology, Şifa University Medicine School, İzmir, Turkey; \\ ${ }^{3}$ Specialist in Gynecology and Obstetrics - Department of Gynecology and Obstetrics, Şifa University \\ Medicine School, İzmir, Turkey.
}

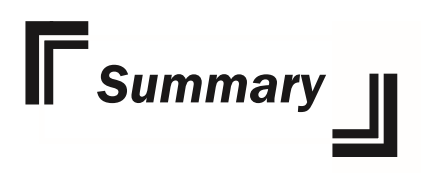

Surgical treatment of female stress urinary incontinence (SUI) has become very popular after respectable success with minimal invasive surgeries. This is the first report of long term vaginocutaneous fistula (VCF) plus inguinal abcess after tension-free vaginal tape (TVT). A 67 year-old woman with vaginal discharge lasting more than 3 years complained with a painful swelling in the left inguinal area for the last three months. She had a medical history of TVT sling procedure for SUI six years ago. She had no history of pelvic surgery, cancer treatment or pelvic irradiation before or after TVT sling. No urethrovaginal or vesicovaginal fistula was found in physical examination and cystocopy. MRI showed a vaginocutenaous fistula and inguinal abcess. This case highlights the need for a high index of suspicion for VCF after TVT.

KEY WORDS: Abcess; Fistula; Tension-free vaginal tape; TVT; Vaginocutaneous.

\section{INTRODUCTION}

Surgical treatment of female stress urinary incontinence (SUI) has become very popular after respectable success with minimal invasive surgeries.

However synthetic materials used in slings has some problems through their biocompatibility. Among the different types of mesh, it seems they induce an inflammatory response (1).

Although tension-free vaginal tape (TVT) has been routinely used to treat female SUI with a high success rate, there are concerns regarding its operative safety in relation to bowel and major blood vessel injuries, bladder and urethral perforation, and postoperative voiding difficulties. Also, urethrovaginal and vesicovaginal fistulas were seen as a result of vaginal erosion leaded by mesh reaction.

We documented a case presented 6 years after TVT sling operation with vaginocutenous fistula (VCF) and inguinal abcess. This is the first report of long term VCF plus inguinal abcess after propylene monofilament sling placement.

\section{Case}

A 67 year-old woman was referred to our Urology clinic with vaginal discharge lasting more than 3 years and sometimes accompanying vaginal bleeding. For the last three months, a painful swelling in the left inguinal area was added to symptoms. She had a medical history of TVT sling procedure for SUI six years ago. She had no history of pelvic surgery, cancer treatment or pelvic irradiation before or after TVT sling.

In the physical examination, painful and hyperemic subcutaneous solid mass was present in the left inguinal area with $3 \times 4 \mathrm{~cm}$ size. In the vaginal examination, there were two solid mass with pedicle in size of $4 \times 4 \mathrm{~cm}$ which was originated from vaginal wall on both paraurethral areas seems like as entrance of sling trocars. Both in vaginal or cystoscopic examination there was no urethravaginal or vesicovaginal fistula. MRI showed a fistula tract originating from vagina to subcutaneous tissue of left inguinal area and mesh materials of TVT (Figure 1A-C).

Both masses in the paraurethral areas were excised through vaginal approach at lithotomy position under 
Figure 1.

MRI Images of vaginocutaneous fistula and abcess.

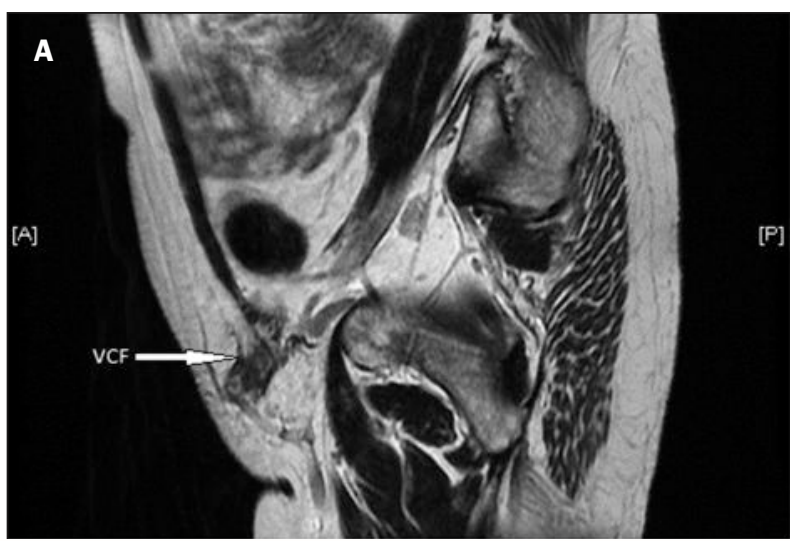

1A: Saggital axis of the VCF.

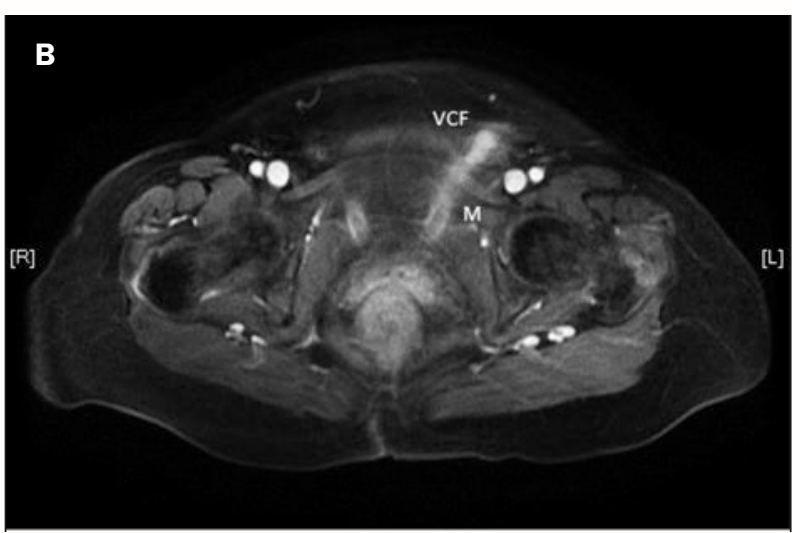

1B: Transver axis, bilateral mesh and left VCF.

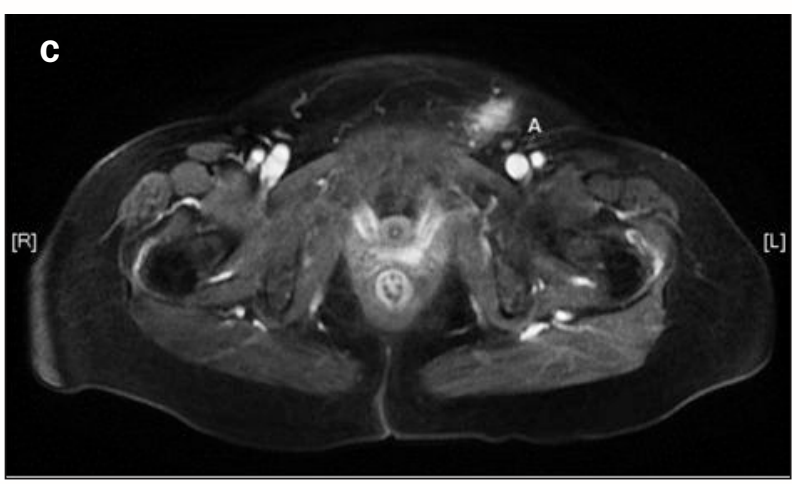

1C: Transver axis, left inguinal abcess.

general anaesthesia. Then, patient was switched to supine position and left inguinal incision was done. Granulation tissue and abcess in the subcutenous tissue was excised. The sling mesh was dissected and removed from the fistula tract. Fistula tract was removed lastly (Figure 2A, B). Skin and subcutaneous tissue was closed. She was discharged after removing urethral catheter and vaginal tampon at the first post operative day. Clinical examination 6 weeks after procedure showed a perfect healing and no SUI.
Figure 2.

Images of fistula tract and TVT mesh after excision.

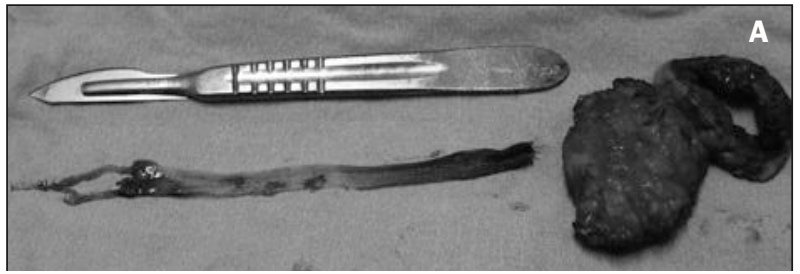

2A: Mesh and inguinal abcess.

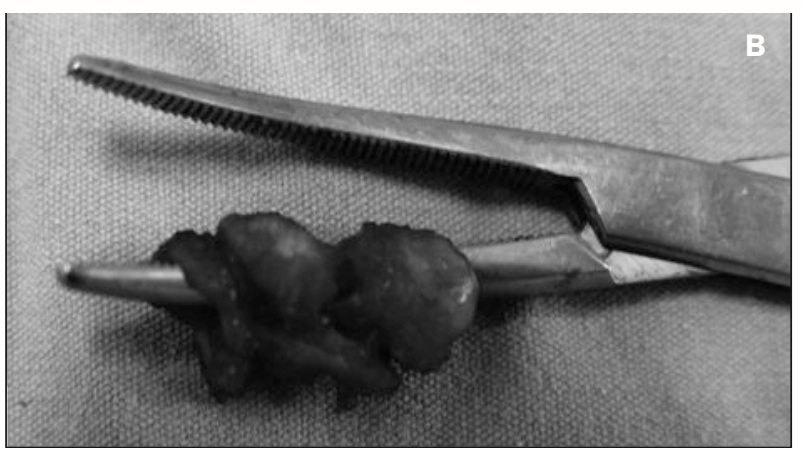

2B: Distal part of fistula tract.

\section{Discussion}

Tension-free vaginal tape is largely performed in the surgical treatment of SUI as a minimal invasive treatment. The TVT procedure has been shown to have a cure rate of $90 \%$ and a low risk of complications (2). Although TVT is minimally invasive surgical procedure, the blind passage of sharp trocars containing risk of injuries. Also mesh has a risk of some inflammatory events because of foreign body reaction.

The risk factors associated with genitourinary fistula formation are the same factors that predispose mesh erosion and include a history of pelvic surgery, pelvic irradiation or history or presence of cancer or infection (2). But in our case there was no history of these risk factors. Also, quite long period was present between the TVT sling procedure and presentation of complications. It may depend on the ignorance of patient or very late reaction of body to mesh.

Urethrovaginal fistula is seen often after complication of pelvic floor surgery that is iatrogenic in developing countries. Since TVT was commonly performed, many reports with urethrovaginal fistulas after TVT were published $(2,3)$. But there is no report of VCF after TVT. Maffiolini and Asteria (4) reported a 64 year-old woman patient with VCF 3 years after Trans-Obturator Tape (TOT) sling. Patient had pre-obturatory abcess surfaced at the prepubic space of tape entry. Firstly mesh was removed after tape exposure was found on the left side of vaginal wall. Two months later pre-obturatory abcess was drained. The patient was referred to authors after worsening of symptoms, and they detected a VCF. Marques et al. (1) reported 54-year-old woman with perineal cellulitis after TOT sling. They have detected subu- 
rethral erosion allowed to see the tape. They removed the tape and infectious tissue after beginning intravenous antibiotic therapy. Marsh and Rogerson (5) reported a 46-year-old woman with groin abcess after TOT erosion. The symptoms started with vaginal discharge 8 weeks after TOT sling. They removed the tape, infectious and necrotic tissue after beginning antibiotic therapy. But their case was not late presentation and seems as an infectious complication. The patient had uncontrolled diabetes which could lead immune deficiency in her medical history.

Estevez (3) reported different pathologic mechanisms might affect due to presentation terms of fistula. They stated that short-term fistulas are probably due to an unknown intra-operative urethral injury or to an excessive tensioning of the sling, whereas, long-term fistulas, diagnosed after several months, may have a different complex physiopathology.

Vaginocutaneous fistula is a recognized, but rare, complication of TVT. This case highlights the need for a high index of suspicion for VCF after TVT. Vaginal examination to identify any vaginal erosion and fistula is crucial in the follow-up of slings. Most complications after slings are underreported. The management of these complications is including removal of all tape, infectious tissue and, fistula tract, and also adjuvant antibiotic therapy.

\section{REFERENCES}

1. Marques AL, Aparício C, Negrão L. Perineal cellulitis as a late complication of trans-obturator sub-urethral tape, Obtape. Int Urogynecol J Pelvic Floor Dysfunct. 2007; 18:821-2.

2. Lowman J, Moore RD, Miklos JR. Tension-free vaginal tape sling with a porcine interposition graft in an irradiated patient with a past history of a urethrovaginal fistula and urethral mesh erosion: a case report. J Reprod Med. 2007; 52:560-2.

3. Estevez JP, Cosson M, Boukerrou M. An uncommon case of urethrovaginal fistula resulting from tension-free vaginal tape. Int Urogynecol J. 2010; 21:889-91.

4. Maffiolini M, Asteria CR. A cutaneous-vaginal fistula and myositis of the obturator muscle following placement of a trans-obturator tape for stress incontinence. Eur J Obstet Gynecol Reprod Biol. 2010; 149:225-6

5. Marsh F, Rogerson L. Groin abscess secondary to trans obturator tape erosion: case report and literature review. Neurourol Urodyn. 2007; 26:543-6.

\section{Correspondence}

Ali Feyzullah Sahin, MD, FEBU

Şifa Üniversitesi Bornova Uygulama ve Araştırma

Hastanesi, Sanayi Cad. No: 735100

Bornova, Izmir, Turkey

uroali@yahoo.com 